\title{
Inhibitory effect of PEG-5-Fu-MAMS combined with constant magnetic field on the growth of LoVo colon cancer cells in vitro.
}

\author{
Jiangang Liu ${ }^{1}$, Zhichao Liu ${ }^{2 *}$, Keqiang Wang ${ }^{3 *}$ \\ ${ }^{1}$ Departments of Surgery, Affiliated Hospital of Taishan Medical University, Tai'an, Shandong Province, PR China \\ ${ }^{2}$ Clinical College of Taishan Medical University, Tai'an, Shandong Province, PR China \\ ${ }^{3}$ Departments of Clinical Laboratory, Affiliated Hospital of Taishan Medical University, Tai'an, Shandong Province, PR \\ China
}

\begin{abstract}
Objective: To investigate the inhibitory effect of polyethylene glycol modified magnetic 5-fluorouracil albumin microspheres (PEG-5-FU-MAMS) combined with external constant magnetic fields on LoVo colon cancer cells in vitro and the mechanism.

Methods: The PEG-5-FU-MAMS were prepared by emulsification/solidification method. The cultured LoVo cells were divided into four groups. Group A was PEG-5-FU-MAMS combined with external magnetic fields; group B was pure PEG-5-FU-MAMS without magnetic fields; group C was free 5fluorouracil (5-FU); group D was pure magnet. The growth of LoVo cells were observed under an inverted microscopy and the inhibitory rate (IR) was assayed by methyl thiazol tetrazolium (MTT) assay.

Results:After different treatment, the tumor growth showed no difference on tumor cells in pure magnet group. The IR of pure PEG-5-FU-MAMS and free 5-FU at same drug concentration was similar, there was no difference between them. Compared with pure PEG-5-FU-MAMS and free 5-FU, when the concentration of 5-FU exceeded $10.0 \mathrm{mg} / \mathrm{L}$, IR was increased after PEG-5-FU-MAMS was combined with constant external magnetic fields.

Conclusion:The growth inhibiting action was significantly enhanced after PEG-5-FU-MAMS combined with constant external magnetic fields.
\end{abstract}

Keywords: Fluorouracil, Albumins, Microspheres, LoVo cells, Constant magnetic field.

\section{Abbreviations}

PEG-5-FU-MAMS: Polyethylene Glycol Modified Magnetic 5-Fluorouracil Albumin Microspheres; 5-FU: 5-Fluorouracil;

MTT: Methyl Thiazol Tetrazolium; IR: Inhibitory Rate; SEM: Standard Electronic Modules.

Accepted on November 16, 2018

\section{Introduction}

Currently, chemotherapy for colon cancer is mostly based on 5(5-FU) based chemotherapy, but its half-life in vivo is short and the removal rate is rapid, and the effective blood concentration cannot be maintained in vivo. Pegylated 5-fluorouracil magnetic albumin microspheres (PEG-5-FU-MAMS) with high load capacity, better magnetic response and sustained release, opens up a new route of administration to the tumor targeted therapy [1]. With the development of tumor targeted therapy and magnetic biology, the progress of using anticancer drugs combined with magnetic field to treat tumor has been improved recently $[2,3]$. Studies have shown that the magnetic field can affect the viability and differentiation of most cells in varying degrees, but the specific proliferative effects are reported differently. This experiment used methyl thiazol tetrazolium (MTT) assay to observe the inhibitory effect and mechanism of PEG-5-FU-MAMS combined with external magnetic fields on LoVo colon cancer cells in vitro, in order to provide a new way for the treatment of colon cancer.

\section{Method}

\section{Materials}

5-Fluorouracil (5-FU); the purity of 99\% (Acros, Belgium); high glucose DMEM (Hyclone, USA); trypan blue, two dimethyl sulfoxide (DMSO), MTT (Sigma, USA); heat inactivated calf serum (GIBCO, USA); Multiskan MK3 eliasa (Shanghai analytical instrument factory); LoVo cells, a human colon cancer cell line (provided by General Surgery Institute of Tianjin Medical University). The constant magnet is designed by Beijing Xinhai air technology development center, which is 
long cubic $8 \mathrm{~cm} \times 5 \mathrm{~cm} \times 5 \mathrm{~cm}$. The surface of the magnetic field strength is $1500 \mathrm{GS}$ and cell culture plate can be placed directly above the magnet together into the incubator.

\section{PEG-5-FU-MAMS preparation and properties}

According to [1], using emulsified curing method, pegylated 5fluorouracil magnetic albumin microspheres (PEG-5-FUMAMS) were prepared as follows: $250 \mathrm{mg} \mathrm{BSA,} 50 \mathrm{mg}$ PEG, $50 \mathrm{mg} \mathrm{5-Fu}$ and $150 \mathrm{mg}$ magnetic fluid were added to $1.5 \mathrm{ml}$ of distilled water. The resulted dispersion was then added dropwise (20 drops/min) into $100 \mathrm{ml}$ of vegetable oil with continuously stirring for $30 \mathrm{~min}$. The resultant emulsion was then denatured by being heated to $160^{\circ} \mathrm{C}$ for $20 \mathrm{~min}$. The suspension was then cooled to $25^{\circ} \mathrm{C}$, washed three times with ether and three times with distilled water. The washed MS were freeze-dried for further experiment; the morphology and particle size were characterized by scanning electron microscopy and Malvern particle size analyzer; alkali digestion at $265 \mathrm{~nm}$ wavelength was determined by calculation of loading; dissolved in $0,5,10,20,30,40,60,90$ min measured the absorbance and study its stability and magnetic response; experimental study on sustained release microspheres drug release in vitro. Characterization of PEG-5-FU-MAMS as following: the average diameter of microspheres is $(1.32 \pm$ $0.50 \mu \mathrm{m}$ ), which is spherical and has a smooth surface (Figure $1)$. The drug loading was $(5.31 \% \pm 0.13 \%)$; had better magnetic response (Figure 2); the release rate of $0.5 \mathrm{~h}$ was $(28.50 \% \pm 0.66 \%)$; the release rate of $24 \mathrm{~h}$ was $(46.93 \% \pm$ $2.83 \%)$.

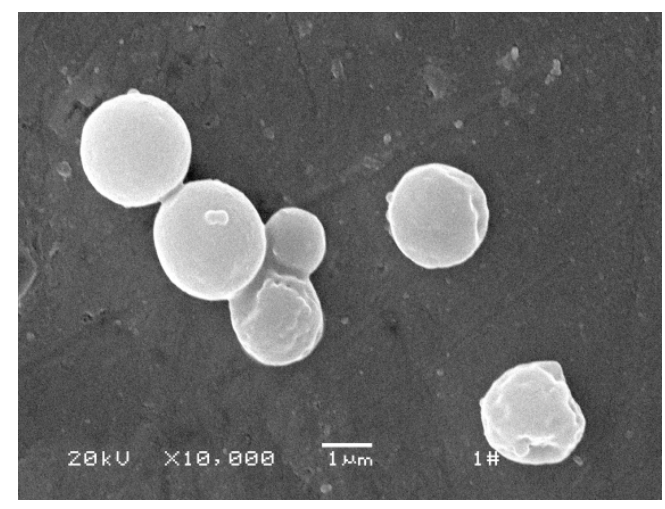

Figure 1. SEM micrograph of PEG-5-Fu-MAMS X10000.

\section{Tumor cell growth inhibition test}

This experiment used methyl thiazol tetrazolium (MTT) assay to observe the inhibitory effect and mechanism of PEG-5-FUMAMS combined with external magnetic fields on LoVo colon cancer cells in vitro. The cultured LoVo cells were divided into four groups. Group A was PEG-5-FU-MAMS combined with external magnetic fields; group B was pure PEG-5-FU-MAMS without magnetic fields; group $\mathrm{C}$ was free 5- fluorouracil (5FU); group D was pure magnet. Referring to the 5-FU plasma peak level of $10 \mathrm{mg} / \mathrm{L}$, each group contained 7 different concentration and microspheres reserved with 5-FU concentration of $1,2,5,10,20,50.0,100.0 \mathrm{mg} / \mathrm{L}$; each condition was repeated with 3 holes. The growth and morphological changes of LoVo cells were observed under an inverted microscopy and the inhibitory rate (IR) was determined by MTT. IR $(\%)=(1-(\mathrm{OD}$ value of experimental group-OD value of blank zero adjustment hole/OD value of control group-OD value of blank zero adjustment hole)) $\times$ $100 \%$; IR $>50 \%$ said the drug sensitive. IR $>50 \%$ said the drug sensitive.

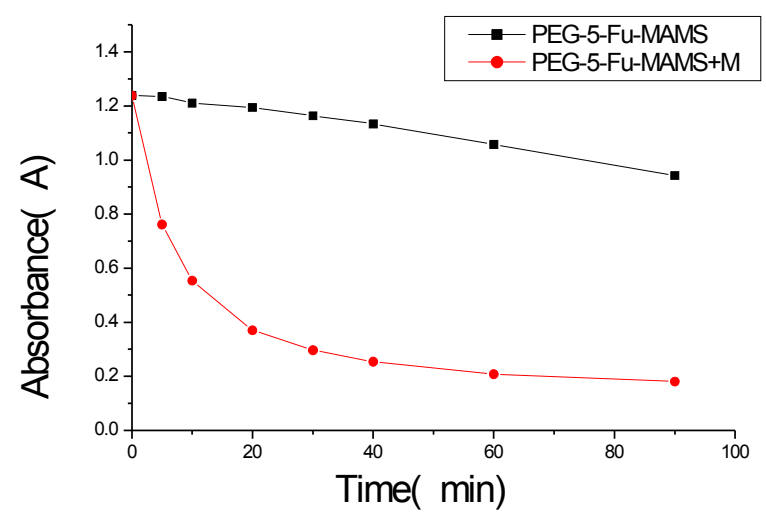

Figure 2. Sedimentation curve of PEG-5-Fu-MAMS.

\section{Statistical analysis method}

The package was analysed by SPSS 13 software, quantitative data with $\overline{\mathrm{x}} \pm \mathrm{s}$, analysis of variance between the 3 groups, the overall average difference, further 22 compared with $\mathrm{Q}$ test, the difference was statistically significant $\mathrm{P}<0.05$.

\section{Results}

LoVo cells attached completely and grew well under the microscope after cultured for $24 \mathrm{~h}$. Cell number per hole is $3 \times$ $10^{4}$. After different treatment, the IR of pure PEG-5-FUMAMS and free 5-FU at same drug concentration was similar, there was no difference between them. Compared with pure PEG-5-FU-MAMS and free 5-FU, IR was increased after PEG-5-FU-MAMS combined with constant external magnetic fields when the concentration of $5-\mathrm{FU}$ exceeded $10.0 \mathrm{mg} / \mathrm{L}$. The drug concentration from $20.0 \mathrm{mg} / \mathrm{L}, \mathrm{A}-\mathrm{C}$ three groups of IR were higher than $50 \%$, drug sensitivity; A group compared with $\mathrm{B}$ group and $\mathrm{C}$ group, there was difference; when the drug concentration is $50.0 \mathrm{mg} / \mathrm{L}$, comparison of three groups of A$\mathrm{C}$, there was difference; when the drug concentration is 100.0 $\mathrm{mg} / \mathrm{L}$, comparison of three groups of A-C, there was difference (Table 1).

After $72 \mathrm{~h}$ continuing culture, we observed that: with the increase of drug concentration, B and C groups, adherent cells gradually reduced, suspended dead cells gradually increased, abnormal morphology, refractive index difference under the microscope; A group showed that adherent cells were significantly less, suspended dead cells increased, morphology was irregular, refraction was poor; LoVo cells of D group adhered well, normal morphology, no obvious inhibitory effect. 

in vitro

When the concentration of 5-FU was $20 \mathrm{mg} / \mathrm{L}$, after culture of $24 \mathrm{~h}, 48 \mathrm{~h}, 72 \mathrm{~h}$ and $96 \mathrm{~h}$, the results showed that the cytotoxicity of PEG-5-FU-MAMS combined with magnetic field on LoVo cells increased gradually with the prolongation of action time, and the inhibition was time-dependent within 96 h. The inhibitory effect of pure PEG-5-Fu-MAMS group and free 5-Fu group on LoVo cells reached the peak at $72 \mathrm{~h}$ and did not increase significantly after $96 \mathrm{~h}$ (Figure 3 ).

Table 1. Inhibition of different concentrations of 5-FU on human colon cancer cell line LoVo rate $(\bar{x} \pm s, \%)$.

\begin{tabular}{|c|c|c|c|c|c|c|c|}
\hline \multirow[t]{2}{*}{ Group } & \multicolumn{7}{|c|}{ Drug concentration (mg/L) } \\
\hline & 1 & 2 & 5 & 10 & 20 & 50 & 100 \\
\hline A & $29 \pm 1$ & $38 \pm 2$ & $42 \pm 2$ & $60 \pm 3$ & $78 \pm 3$ & $81 \pm 4$ & $87 \pm 4$ \\
\hline B & $13 \pm 1$ & $18 \pm 1 a b$ & $26 \pm 2 a b$ & $43 \pm 2$ & $54 \pm 3 a$ & $59 \pm 3 a$ & $64 \pm 3 a$ \\
\hline C & $17 \pm 2 a b$ & $24 \pm 2 a b$ & $28 \pm 2 a b$ & $40 \pm 2 a b$ & $58 \pm 3 a b$ & $56 \pm 3 a b$ & $59 \pm 3 a b$ \\
\hline $\mathrm{F}$ & 126 & 115 & 51 & 62 & 67 & 57 & 66 \\
\hline$P$ & $<0.01$ & $<0.01$ & $<0.01$ & $<0.01$ & $<0.01$ & $<0.01$ & $<0.01$ \\
\hline
\end{tabular}

Note: compared with group A, aP $<0.05$; compared with group B, ${ }^{\mathrm{b}} \mathrm{P}>0.05$. Group A consisted of magnetic 5-fluorouracil albumin microspheres combined magnetic field group, group B was 5- luorouracil albumin microsphere group, group C was free 5-fluorouracil group, and group D was simple magnetic field group.

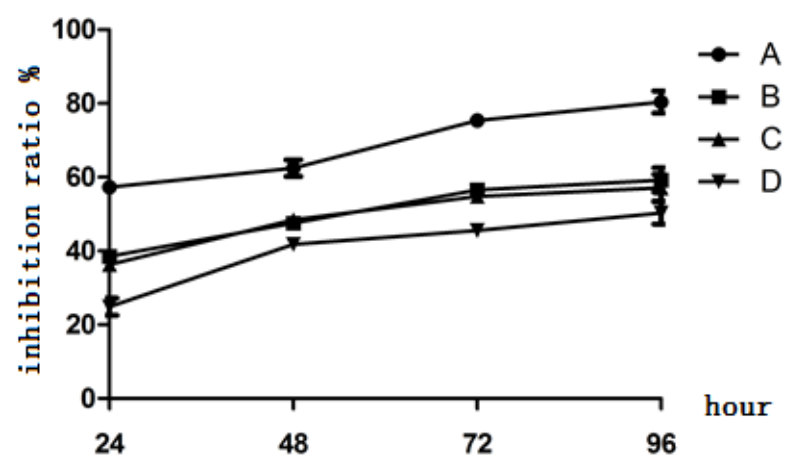

Figure 3. Cytotoxicity of colon cancer cells at different time of action under the influence of 5- fluorouracil concentration of $20.0 \mathrm{mg} / \mathrm{L}$. Group A consisted of magnetic 5-fluorouracil albumin microspheres combined magnetic field group, group B was 5-fluorouracil albumin microsphere group, group C was free 5-fluorouracil group, and group $D$ was simple magnetic field group.

\section{Discussion}

The traditional anti-tumor drugs are treated by various routes, and the blood serum concentration is distributed in the whole body to produce therapeutic effect. The biggest disadvantage of this treatment is lack of selectivity, which often results in severe side effects, and thereby affecting the therapeutic value of these drugs [4]. In order to overcome the disadvantages of traditional medicine on these problems, people put forward a number of targeted drug delivery method, which the medicine package to the magnetic nanoparticles and then controlled by external conditions, effectively achieve the purpose of particle transfer, in vivo aggregation, of disease areas targeted therapy as a method $[5,6]$.

This study found that: the magnetic field group had no obvious effect on the growth of LoVo cells; the IR of pure PEG-5-FUMAMS and free 5-FU at same drug concentration was similar, while PEG-5-Fu-MAMS combined with magnetic field group was significantly increased. The result indicates that magnetic microsphere preparation method and dosage form change had no influence on its pharmacological effect. It also showed that the cytotoxicity to LoVo cells increased with the increase of drug concentration, and the inhibitory effect increased with the prolongation of action time within $96 \mathrm{~h}$ after PEG-5-FuMAMS combined with magnetic field. There was a double dose and time dependence. The combination of the two methods has obvious synergistic killing effect. The mechanism may be due to: (1) Under the action of magnetic field intensity and gradient, magnetic drug-loaded microspheres aggregate in large quantities to form drug storehouse, and local drug concentration increases [7,8]. (2) In a certain period of time, the sustained-release of chemotherapeutic drugs carried by magnetic drug-loaded microspheres can further kill residual cancer cells, making tumor treatment more thoroughly [9]. (3) At the stage of DNA synthesis of tumor cells, when the external magnetic field is applied, the newly synthesized DNA strands which are in a loose state are changed due to the interference of magnetic field, which is more conducive to the killing effect of 5-Fu with DNA as the target [9]. (4) The magnetic field penetrates the cell membrane. Under the action of magnetic field, the trajectory of charged ions on the membrane surface is restrained due to Lorentz force, which leads to the abnormal movement and transmission of electrons and ions on the membrane of tumor cells, resulting in the change of the microstructure of the membrane of tumor cells, thereby affecting the trajectory. The permeability and orderliness of cell membrane [10] provided conditions for faster and more penetration of chemotherapeutics molecules into cell membrane, which may be one of the reasons that magnetic field combined with magnetic chemotherapeutic drugs can significantly improve its tumor inhibition effect. Its specific mechanism needs further study. 


\section{Acknowledgements}

Thanks General Surgery Institute of Tianjin Medical University for providing LoVo cells, a human colon cancer cell line. The authors thank all study-site staff and participating consultants for their contributions, which made this study possible.

\section{Role of the Funding Source}

This study was funded by the National Natural Science Foundation of China (No. 81473687), Shandong medical and health science and technology development plan (No. 2009HW015), Shandong science and technology development plan (No. 2013YD- 21013).

\section{Availability of Data and Materials}

The datasets used and analysed during the current study are available from the corresponding author on reasonable request.

\section{Authors' Contributions}

$\mathrm{JL}$ performed experiments and analysed the statistics. KW were major contributors in writing the manuscript. $\mathrm{ZL}$ designed the experiments and provided funding. All authors read and approved the final manuscript.

\section{Ethics Approval and Consent to Participate}

All procedures performed in studies involving human participants were in accordance with the ethical standards of the institutional and national research committee and with the Helsinki declaration and its later amendments or comparable ethical standards. The study was performed according to the Declaration of Helsinki and was approved by the ethics committee of the affiliated hospital of Taishan University. Written informed consents were obtained from all the subjects recruited into our study.

\section{Competing Interests}

JL, ZL and KW declare that they have no competing interest.

\section{References}

1. Liu JG, Liu ZC, Qian ZY, Sun CY, Wang YZ, Yu C. Preparation and properties in vitro of the PEG modified magnetic albumin microspheres. Zhong Hua Shi Yan Wai Ke Za Zhi 2008; 25: 36-38.

2. Zhang L, Wang JH, Wang HL, Wang WC, Li ZY, Liu JJ, Yang XX, Ji XM, Luo Y, Hu C, Hou YB, He QQ, Fang J,
Wang JF, Liu QS, Li GH, Lu QY, Zhang X. Moderate and strong static magnetic fields directly affect EGFR kinase domain orientation to inhibit cancer cell proliferation. Oncotarget 2016; 7: 41527-41539.

3. Soumaya G, Aida L, Mohsen S, Hafedh A. Bioeffects of static magnetic fields: oxidative stress, genotoxic effects, and cancer studies. Biomed Res Int 2013; 2013: 602987.

4. Liu ZC, Liu JG, Kong X. Comparison of targeting distribution of PEG-5-Fu-MAMS and 5-Fu-MAMS in liver. Zhong Guo Xian Dai Pu Tong Wai Ke Jin Zhan 2010; 13: 594-596.

5. Nasimudeen RJ, Shams T, Ghulam MA, Shazi S, Ghazi AD, Mohammad AK. Nanotechnology-based approaches in anticancer research. Int $\mathrm{J}$ Nanomedicine 2012; 7: 4391-4408.

6. Charalambos K, Travis MS, Daniel LJT, Jan G. Dawn of advanced molecular medicine: nanotechnological advancements in cancer imaging and therapy. Crit Rev Oncog 2014; 19: 143-176.

7. Cui JJ, Jin GY, Xia L, Quan JS. Application of superparamagnetic iron oxide nanoparticles in magnetic resonance imaging and drug delivery system. Guo Ji Yao Xue Yan Jiu Za Zhi 2015; 42: 601-605.

8. Lee PW, Hsu SH, Wang JJ, Tsai JS, Lin KJ, Wey SP, Chen FR, Lai CH, Yen TC, Sung HW. The characteristics, biodistribution, magnetic resonance imaging and biodegradability of superparamagnetic core-shell nanoparticles. Biomaterials 2010; 31: 1316-1324.

9. Anatoly L, Buchachenko, Alexei P, Orlov, Dmitry A, Kuznetsov, Natalia N. Breslavskaya. Magnetic isotope and magnetic field effects on the DNA synthesis. Nucleic Acids Res 2013; 41: 8300-8307.

10. Vitalii Z, Tatyana P, Oleg L, Alexandr D. How a highgradient magnetic field could affect cell life. Sci Rep 2016; 6: 37407.

\section{*Correspondence to}

Zhichao Liu

Clinical College of Taishan Medical University

PR China

Keqiang Wang

Department of Clinical Laboratory

The Affiliated Hospital of Taishan Medical University

PR China 